\title{
Forces on Small Particles in Nonhomogeneous Polyatomic Gases
}

\author{
H. Vestner* \\ Department of Physics, Texas A \& M University, College Station, Texas 77843
}

(Z. Naturforsch. 29 a, 1244-1252 [1974] ; received March 15 th, 1974 )

\begin{abstract}
Waldmann's work on forces on small particles suspended in a nonhomogeneous monatomic gas is extended to a polyatomic gas of rotating molecules. Under the assumption that the coefficient of diffuse reflection is equal to 1 , the force the gas exerts on a particle is calculated from an expansion of the distribution function. In general, this force consists of three contributions which are determined by the flow velocity, the translational heat flux, and the pressure tensor in the gas. The latter force vanishes for spheroidal particles, leaving only the frictional and the thermal force. These two forces are given for prolate and oblate spheroids. In particular, the dependence of the thermal force on the orientation of the particle's axis relative to the temperature gradient is dis. cussed, and a comparison with the hydrodynamic and free molecular results is made.
\end{abstract}

Particles at rest in a streaming and heat conducting gas are subject to forces which are proportional to the flow velocity and the heat flux in the gas, viz. the frictional and the thermal forces, respectively. These forces depend strongly on the ratios of the characteristic lengths of the system, i. e. the size a of the particle, the mean free path $l$ of a gas molecule, and the size $D$ of the macroscopic container. Here, the discussion is restricted to particles which are small on the macroscopic scale, i. e. where $a \ll D$ applies.

With increasing mean free path the frictional force decreases from its pressure independent hydrodynamic value (for $l \ll a$ ) to the free molecular flow limit (for $l \gg a$ ), which is proportional to pressure $p$. For small mean free paths $l$ the frictional force is linear in $a$, for large values of $l$ it is proportional to the surface of the particle, i. e. quadratic in $a$. The most prominent expressions for these limits are the formulas for spheres given by Stokes (for $l \ll a$, see e. g. Lamb ${ }^{1}$ ) and by Epstein ${ }^{2}$ (for $l \gg a$ ). The frictional force for a general ellipsoid is stated in the hydrodynamic limit by Lamb ${ }^{1}$.

The thermal force in the hydrodynamic regime is caused by the thermal slip, hence it is proportional to the mean free path $l$, and increases with decreasing pressure like $p^{-1}$. In the free molecular flow limit $(a \ll D \ll l)$ the thermal force decreases proportional to pressure. And in between (i.e. for $a \ll l \ll D$ ) it reaches its highest pressure independent value, first calculated roughly by Einstein ${ }^{3}$, and more exactly by Waldmann ${ }^{4,5}$. Most calculations

Reprint requests to Dr. H. Vestner, Institut für Theoretische Physik der Universität Erlangen-Nürnberg, D-8520 Erlangen, Glückstrasse 6. have been performed for spheres, namely by Hettner $^{6}$ and Epstein ${ }^{7}(l \ll a \ll D)$, by Einstein ${ }^{3}$ and Waldmann ${ }^{4,5}(a \ll l \ll D)$, and by Brock ${ }^{8}$ and Phillips ${ }^{9}(a \ll D \ll l)$. Expressions for disks in the hydrodynamic and the free molecular flow limits are due to Epstein $^{7}$ and Knudsen ${ }^{10}$, respectively. A hydrodynamic calculation for a prolate spheroid * * has been performed by Sexl ${ }^{11}$, similar to Lamb's treatment ${ }^{1}$ of the frictional force for a general ellipsoid. For small mean free paths $(l \ll a)$ the thermal force is a linear function of the particle size $a$, if $l$ is large compared to $a$ (i.e. for $a \ll l \ll D$, and $a \ll D \ll l$ ) it is proportional to the surface of the particle.

All calculations for the intermediate regions are rather difficult, e. g. in the slip flow regime $(l \ll a$ up to $l \lesssim a$ ) elaborate boundary conditions have to be used. For a comprehensive review of theoretical and experimental work on the thermal force the article by Waldmann and Schmitt ${ }^{12}$ may be consulted. More recent work in the slip flow regime is due to Jacobsen and Brock ${ }^{13}$, Dwyer ${ }^{14}$, and to Vestner, Kübel and Waldmann ${ }^{15}$. The thermal force on a small sphere (radius a) between parallel plates (distance $D$ ) has recently been calculated by Phillips ${ }^{9}$ for $a \ll D$ from $a \leqslant l$ up to $D \ll l$ using a twostream Chapman-Enskog distribution function.

The purpose of this paper is the calculation of the forces on small spheroidal particles suspended in a polyatomic gas of rotating molecules. Specifically $a \ll l$ applies; in addition, for the thermal force, the validity of $a \ll l \ll D$ is assumed, where $D$ is the

\footnotetext{
* Permanent address: Institut für Theoretische Physik der Universität Erlangen-Nürnberg, Erlangen (Germany). ** i. e. an axisymmetric ellipsoid.
} 
distance between parallel plates creating the temperature difference in the gas. In Waldmann's paper ${ }^{4,5}$ a monatomic gas is considered, and the final expressions are evaluated only for spherical particles. The first part of this paper deals with the calculation of the force for general bodies, with the following assumptions:

(1) The presence of the small particle does not disturb the distribution function of the approaching molecules.

(2) All molecules are reflected diffusely from the surface, i. e. the coefficient $\alpha$ of diffuse reflection is put equal to 1 , whereas Waldmann ${ }^{4,5}$ (for monatomic gases) allows for values of $\alpha$ different from 1.

Then the force is calculated from a special expansion of the distribution function within the gas. For many polyatomic gases this expansion provides a good approximation for the treatment of flow and heat conduction in the presence of a magnetic field, i. e. for the Senftleben-Beenakker effect ${ }^{16}$. The total force on the particle is determined by only three of the nine expansion coefficients, namely by the flow velocity $\boldsymbol{v}$, the translational heat flux $q_{\text {trans }}$ and the symmetric traceless part $\overline{\mathbf{p}}$ of the pressure tensor. This last type of force is only present if $\overline{\mathbf{p}}$ is nonzero, and if unsymmetric bodies are involved, like a hemisphere. For spheroidal particles only the frictional and the thermal forces remain. Explicit expressions for prolate and oblate spheroids are given.

The magnetic field dependent part of the translational heat flux gives rise to the thermomagnetic force, which has been calculated previously for spheres by Hess ${ }^{17}$. Measurements of this magnetic field induced change in the thermal force on disks have been performed by Adair and coworkers ${ }^{18}$. The thermomagnetic force for spheroids will be discussed in detail in a subsequent paper.

The second part of this paper is devoted to the discussion of the frictional and thermal forces for zero magnetic field. Special attention is given to flat disks and long rods, to the dependence of the thermal force on the orientation of these particles relative to the temperature gradient, and to the comparison with the corresponding results in the hydrodynamic and free molecular flow limits.

Finally, in the Appendix, the hydrodynamic expressions for the frictional ${ }^{1}$ and for the thermal force (partly due to Sexl ${ }^{11}$ ) are given, and the ex- plicit formulas for prolate and oblate spheroids (see e. g. Ref. ${ }^{19}$ ) are stated.

\section{Calculation of the Force}

\section{A General Formula}

A small body with a surface $\sigma$ is immersed in a polyatomic gas of rotating molecules. The force on the surface element $\mathrm{d} \sigma$ with the outer unit normal $\boldsymbol{n}$ is given by the momentum transfer from the gas:

$$
\begin{aligned}
\mathrm{d} \boldsymbol{K}=-\left[\operatorname{Tr} \int m \boldsymbol{c}\right. & c_{n} f_{j}^{-} \mathrm{d}^{3} c \\
& \left.+\operatorname{Tr} \int_{+} m \boldsymbol{c} c_{n} f_{j}^{+} \mathrm{d}^{3} c\right] \mathrm{d} \sigma .
\end{aligned}
$$

Here, $\boldsymbol{c}$ is the molecular velocity, $c_{n}=\boldsymbol{c} \cdot \boldsymbol{n}$ its normal component, and $m$ is the molecular mass. The distribution function for molecules in the rotational state $j$ is denoted by $f_{j}$, consequently $f_{j}^{-}$describes the molecules approaching the particle $\left(c_{n}<0\right)$ and $f_{j}{ }^{+}$the molecules reflected from the surface $\left(c_{n}>0\right)$. The subscripts \pm on the integrals indicate integration over $c_{n}>0$ and $c_{n}<0$, respectively; the operation $\mathrm{Tr}$ includes the trace over magnetic quantum numbers and a summation over $j$.

Since the particle is much smaller than the mean free path $l$ of a gas molecule, it can be assumed that the distribution function of the approaching molecules is not disturbed by the presence of the particle $^{4}$, i. e.

$$
f_{j}^{-}=f_{j} .
$$

For $f_{j}{ }^{+}$the assumption is made, that all molecules are reflected diffusely with a Maxwell distribution

$$
f_{j}^{+}=f_{j}^{\mathrm{MI}}\left(n^{+}, T_{\mathrm{t}}{ }^{+}, T_{\mathrm{r}}{ }^{+}\right) .
$$

Since the coefficient $\alpha$ of diffuse reflection is put equal to 1 , molecules are reflected with random orientation of their angular momenta. Incomplete accommodation of translational and rotational energy is taken into account by allowing $T_{\mathrm{t}}{ }^{+}$and $T_{\mathrm{r}}{ }^{+}$to be different from the temperature of the particle. All three temperatures are assumed to be constant over the surface $\sigma$ of the particle. In

$$
\begin{gathered}
f_{j}^{\mathrm{M}}\left(n^{+}, T_{\mathrm{t}}^{+}, T_{\mathrm{r}}^{+}\right)=n^{+}\left(m / 2 \pi k T_{\mathrm{t}}^{+}\right)^{3 / 2} Q_{\mathrm{r}}^{-1} \\
\quad \exp \left\{-\left(m c^{2} / 2 k T_{\mathrm{t}}^{+}\right)-\left(E_{j} / k T_{\mathrm{r}}^{+}\right)\right\},
\end{gathered}
$$

$k$ is the Boltzmann constant, $E_{j}=\hbar^{2} j(j+1) / 2 \Theta$ is the rotational energy of a molecule (in the state $j$ ) with the moment of inertia $\Theta$, and

$$
Q_{\mathrm{r}}=\sum_{j^{\prime}}\left(2 j^{\prime}+1\right) \exp \left\{-E_{j}^{\prime} / k T_{\mathrm{r}}{ }^{+}\right\}
$$


is the rotational partition sum. The density $n^{+}$has to be determined such that no molecules accumulate at the surface ${ }^{4}$ :

$$
\operatorname{Tr} \int_{-} c_{n} f_{j}^{-} \mathrm{d}^{3} c+\operatorname{Tr} \int_{+} c_{n} f_{j}^{+} \mathrm{d}^{3} c=0 .
$$

If $\Phi_{j}$ denotes the deviation of $f_{j}$ from its equilibrium value $f_{j}{ }^{0}=f_{j}^{\mathrm{M}}\left(n_{0}, T_{0}, T_{0}\right)$ we have

$$
f_{j}=f_{j}^{0}\left(1+\Phi_{j}\right) \text {. }
$$

Then, by use of Eqs. (1.2), (1.3), and of the relation

$$
\operatorname{Tr} \int_{ \pm} c_{n} f_{j}^{\mathrm{M}}\left(n^{+}, T_{\mathrm{t}}^{+}, T_{\mathrm{r}}^{+}\right)= \pm \frac{1}{4} n^{+} \sqrt{8} k T_{\mathrm{t}}^{+} / \pi m,
$$

Eq. (1.5) can be solved for $n^{+}$:

$$
n^{+}=n^{0}\left(V T_{0} / T_{\mathrm{t}}^{+}+X\right) .
$$

Here, $X$ is given by

$$
X \equiv-2 \sqrt{\tau} \operatorname{Tr} \int \beta_{0}{ }^{1 / 2} c_{n}\left(f_{j}{ }^{0} / n_{0}\right) \Phi_{j} \mathrm{~d}^{3} c,
$$

with $\beta_{0}=m / 2 k T_{0}$. For small deviations from equilibrium Eq. (1.7) can be replaced by

$$
n^{+}=n^{0}\left(V T_{0} / T_{\mathrm{t}}^{+}+X\right) .
$$

The temperatures $T_{\mathrm{t}}{ }^{+}$and $T_{\mathrm{r}}{ }^{+}$depend on the temperature of the particle and on the state of the gas. They are not calculated here since they do not contribute to the force

$$
\boldsymbol{K}=-\frac{1}{2} p_{\jmath} \iint \mathrm{d} \sigma(\boldsymbol{X}+\boldsymbol{n} X) .
$$

By $p_{0}=n_{0} k T_{0}$ the equilibrium gas pressure is denoted, and $\boldsymbol{X}$ is an abbreviation for

$$
\boldsymbol{X}=4 \operatorname{Tr} i \beta_{0} \boldsymbol{c} c_{n}\left(f_{j}^{0} / n_{0}\right) \Phi_{j} \mathrm{~d}^{3} c .
$$

In deriving Eq. (1.9) from Eqs. (1.1) - (1.3) use has been made of the relation

$$
\operatorname{Tr} \int_{ \pm} m \boldsymbol{c} c_{n} f_{j}^{\mathrm{MI}}\left(n^{+}, T_{\mathrm{t}}^{+}, T_{\mathrm{r}}^{+}\right) \mathrm{d}^{3} c=\frac{1}{2} n^{+} k T_{\mathrm{t}}^{+} \boldsymbol{n},
$$

of Eq. $\left(1.7^{\prime}\right)$ and of

$$
\iint \mathrm{d} \sigma \boldsymbol{n}=0 .
$$

The general formula Eq. (1.9) can only be evaluated if the quantities $X$ and $\boldsymbol{X}$ are known, i. e. if the nonequilibrium state of the gas, as described by $\Phi_{j}$, is specified.

\section{A Special Expansion of the Distribution Function}

By application of the moment method ${ }^{20}$, a system of coupled differential equations for mean values can be derived from the generalized Boltzmann equa- tion due to Waldmann and Snider ${ }^{21}$. To this end the distribution function is expanded into a complete set of symmetric traceless tensors $\Phi_{\mu_{1} \ldots \mu_{l}}^{(k)}$ built up from the dimensionless molecular velocity $\boldsymbol{C}=\beta_{0}{ }^{1 / 2} \boldsymbol{c}$ and the rotational angular momentum $\boldsymbol{J}$. Here this expansion is restricted to the finite set of tensors which has proven to be sufficient for a description of the magnetic field dependent flow and heat conduction in most polyatomic gases, i. e. for the SenftlebenBeenakker effect ${ }^{16}$ :

$\Phi_{j}=P^{j} \Phi P^{j}, \Phi=\sum_{l=0}^{2} \sum_{k=1}^{N_{1}} \Phi_{\mu_{1} \ldots \mu_{l}}^{(k)} a_{\mu_{1} \ldots \mu_{l}}^{(k)}+\Psi_{k, \mu \nu} b_{i, \mu \nu}$, $N_{0}=N_{1}=3, N_{2}=2$.

$P^{j}$ is a projection operator on the $j$-subspace ${ }^{20}$. Differential equations for the expansion coefficients $a_{\mu_{1} \ldots \mu_{t}}^{(k)}$ are obtained ${ }^{20}$ by taking moments of the Waldmann-Snider equation. The moments occurring in Eq. (2.1) are related to the following physical quantities: the density $n$, the translational and rotational temperatures $T_{\text {trans }}$ and $T_{\text {rot }}$, the corresponding heat fluxes $\boldsymbol{q}_{\text {trans }}$ and $\boldsymbol{q}_{\text {rot }}$, the flow velocity $\boldsymbol{v}$, the frictional pressure tensor $\overline{\mathbf{p}}$, the tensor polarization $\mathbf{a}^{(2)}$ and the flux of tensor polarization $\underline{\mathbf{b}}$. The two quantities $\mathbf{a}^{(2)}$ and $\underline{\mathbf{b}}$ are polarizations set up by collisions. They depend on a magnetic field, and due to the collisional coupling with $\overline{\mathbf{p}}, \boldsymbol{q}_{\text {trans }}$ and $\boldsymbol{q}_{\text {rot }}$ they account for the field effects of viscosity and thermal conductivity ${ }^{16}$.

Due to the relations

$$
\operatorname{Tr}\left(\varepsilon-\langle\varepsilon\rangle_{0}\right) \exp \{-\varepsilon\}=0, \operatorname{Tr} \overline{\boldsymbol{J} J} \exp \{-\varepsilon\}=0
$$

the tensors

$$
\begin{aligned}
\Phi^{(3)} & \propto \varepsilon-\langle\varepsilon\rangle_{0}, & \boldsymbol{\Phi}^{(3)} & \propto \boldsymbol{C}\left(\varepsilon-\langle\varepsilon\rangle_{0}\right), \\
\boldsymbol{\Phi}^{(2)} & \propto \bar{J} \boldsymbol{J}, & \Psi & \propto \boldsymbol{C} \boldsymbol{\Phi}^{(2)}
\end{aligned}
$$

give no contribution to the force; $\varepsilon=\hbar^{2} J^{2} / 2 \Theta k T_{0}$, $\langle\ldots\rangle_{0}$ refers to an equilibrium average. The tensors and moments relevant for the quantities $X$ and $\boldsymbol{X}$ are then:

$\Phi^{(1)}=1, a^{(1)}=\left(n-n_{0}\right) / n_{0}$,

$\Phi^{(2)}=\sqrt{\frac{2}{3}}\left(\mathbf{C}^{2}-\frac{3}{2}\right), a^{(2)}=\sqrt{\frac{3}{2}} \frac{T_{\text {trans }}-T_{0}}{T_{0}}$,

$\boldsymbol{\Phi}^{(1)}=\sqrt{ } 2 \boldsymbol{C}, \boldsymbol{a}^{(1)}=\sqrt{ } 2 \beta_{0}{ }^{1 / 2} \boldsymbol{v}$, $\boldsymbol{\Phi}(2)={ }_{\sqrt{5}} \boldsymbol{C}\left(\mathrm{C}^{2}-\frac{5}{2}\right), \boldsymbol{a}^{(2)}=\sqrt{\frac{2}{5}} \beta_{0}{ }^{1 / 2} p_{0}{ }^{-1} \boldsymbol{q}_{\text {trans }}$,

$\Phi^{(1)}=\sqrt{2} \overline{\boldsymbol{C}}, \mathbf{a}^{(1)}=\left(\sqrt{2} p_{0}\right)^{-1} \overline{\mathbf{p}}$.

The symbol $\ldots$ indicates the symmetric traceless part of a tensor. 


\section{The Force}

The contributions of these five tensors to $X$ and $\boldsymbol{X}$ in Eqs. (1.8), (1.10) can easily be calculated, see e. g. the Appendix of Ref. 5 for the half-space integrals :

$$
\begin{aligned}
X=\frac{n-n_{0}}{n_{0}}+\frac{1}{2} & \frac{T_{\text {trans }}-T_{0}}{T_{0}} \\
& -\frac{2}{\bar{c}} \boldsymbol{n} \cdot \boldsymbol{v}+\left(2 p_{0}\right)^{-1} \boldsymbol{n} \boldsymbol{n}: \overline{\mathbf{p}},
\end{aligned}
$$

$\boldsymbol{X}=\boldsymbol{n}\left(\frac{n-n_{0}}{n_{0}}+\frac{T_{\text {trans }}-T_{0}}{T_{0}}\right)$

$-\frac{4}{\bar{\tau} c}(\boldsymbol{n} \boldsymbol{n}+\boldsymbol{\delta}) \cdot\left(\boldsymbol{v}+\frac{1}{5 p_{0}} \boldsymbol{q}_{\mathrm{trans}}\right)+p_{0}{ }^{-1} \boldsymbol{n} \cdot \overline{\mathbf{p}}$.

Here $\delta$ is the unit tensor, and $\bar{c}=\sqrt{8 k T_{0}} / \pi m$ is the mean molecular velocity. By integration over the surface, cf. Eq. (1.9), all terms linear in the normal $\boldsymbol{n}$ vanish, thus the force

$$
\begin{aligned}
\boldsymbol{K}=p_{0} \iint \mathrm{d} \sigma & {\left[\frac{2}{\pi \bar{c}}(\boldsymbol{n} \boldsymbol{n}+\delta) \cdot\left(\boldsymbol{v}+\frac{1}{5 p_{0}} \boldsymbol{q}_{\text {trans }}\right)\right.} \\
& \left.+\frac{1}{c} \boldsymbol{n} \boldsymbol{n} \cdot \boldsymbol{v}-\frac{1}{4 p_{0}} \boldsymbol{n}(\boldsymbol{n} \boldsymbol{n}: \overline{\mathbf{p}})\right]
\end{aligned}
$$

is determined by only three moments, namely the flow velocity $\boldsymbol{v}$, the translational heat flux $\boldsymbol{q}_{\text {trans }}$ and the pressure tensor $\overline{\mathbf{p}}$.

The integrals $\iint \mathrm{d} \sigma \boldsymbol{n} \boldsymbol{n}$ and $\iint \mathrm{d} \sigma \boldsymbol{n} \boldsymbol{n}$ are characteristic for the surface of the particle. Whereas the second rank tensor is nonzero for all types of bodies (its trace gives the surface area), the third rank tensor vanishes for highly symmetric bodies, e. g. for spheroids. For a circular cylinder of length $L$ and radius $a$ with one spherical cap (i.e. a hemisphere for $L=0$ ) one has:

$$
\iint \mathrm{d} \sigma=2 \pi a L+3 \pi a^{2},
$$

$\iint \mathrm{d} \sigma \boldsymbol{n} \boldsymbol{n}=\boldsymbol{\delta}\left(\pi a L+\frac{2}{3} \pi a^{2}\right)+\boldsymbol{s} \boldsymbol{s}\left(\pi a^{2}-\pi a L\right)$, $\iint \mathrm{d} \sigma n_{\mu} n_{\nu} n_{\lambda}=\frac{1}{4} \pi a^{2}\left[s_{\mu} \delta_{\nu \lambda}+s_{\nu} \delta_{\mu \lambda}+s_{\lambda} \delta_{\mu \nu}-5 s_{\mu} s_{\nu} s_{\lambda}\right]$;

$\boldsymbol{s}$ is the unit vector parallel to the cylinder axis pointing towards the circular cap. If such a particle is placed in a plane Couette flow the existence of the pressure tensor $\overline{\mathbf{p}}$ leads to small corrections in the frictional force of the order $l / D(D$ is the plate spacing). A method for creating a nonzero value of $\overline{\mathbf{p}}$ in a stagnant polyatomic gas has been pointed out by $\mathrm{Hess}^{22}$. If a tensor polarization is maintained externally (e.g. by optical pumping), a pressure tensor $\overline{\mathbf{p}}$ is created due to collisions. This could give rise to a force on a nonsymmetric body.
For spheroidal particles, however, the total force

$$
\boldsymbol{K}=\boldsymbol{K}^{\mathrm{v}}+\mathbf{K}^{\text {th }}
$$

consists only of the frictional force

$$
\begin{aligned}
\boldsymbol{K}^{\mathbf{v}} & =k^{\|} \boldsymbol{s} \boldsymbol{s} \cdot \boldsymbol{V}+k^{\perp}(\boldsymbol{\delta}-\boldsymbol{s} \boldsymbol{s}) \cdot \boldsymbol{V}, \boldsymbol{V}=(1 / \bar{c}) \boldsymbol{v}, \\
k^{\|} & =p_{0} F(2 / \pi)[1+(1+\pi / 2)(1-2 N)], \\
k^{\perp} & =p_{0} F(2 / \pi)[1+(1+\pi / 2) N],
\end{aligned}
$$

and of the thermal force

$$
\begin{gathered}
\boldsymbol{K}^{\text {th }}=f^{\|} \boldsymbol{s} \boldsymbol{s} \cdot \boldsymbol{Q}+f^{\perp}(\boldsymbol{\delta}-\boldsymbol{s} \boldsymbol{s}) \cdot \boldsymbol{Q}, \boldsymbol{Q}=(1 / \bar{c}) \boldsymbol{q}_{\text {trans }}, \\
f^{\|}==F(4 / 5 \pi)(1-N), f^{\perp}=F(2 / 5 \pi)(1+N) .
\end{gathered}
$$

Here, $s$ denotes a unit vector parallel to the symmetry axis, $F$ is the surface area of the particle, and $N$ is defined by

$$
(1 / F) \iint \mathrm{d} \sigma \boldsymbol{n} \boldsymbol{n}=N \boldsymbol{\delta}+(1-3 N) \boldsymbol{s} \boldsymbol{s},
$$

i. e. $1-3 N$ characterizes the deviation of the particle from the sphere. If $a_{\|}$and $a_{\perp}$ denote the semi-axis parallel and perpendicular to $\boldsymbol{s}$ respectively, the quantities $F$ and $N$ can be written as

$$
\begin{gathered}
F=2 \pi a_{\perp}^{2}+\pi \frac{a^{2}}{\varepsilon} \ln \frac{1+\varepsilon}{1-\varepsilon}, \\
N=\frac{1-\varepsilon^{2}}{2 \varepsilon^{2}}\left[\frac{1+\varepsilon^{2}}{2 \varepsilon} \ln \frac{1+\varepsilon}{1-\varepsilon}-1\right] \\
\cdot\left[\frac{1-\varepsilon^{2}}{2 \varepsilon} \ln \frac{1+\varepsilon}{1-\varepsilon}+1\right]^{-1},
\end{gathered}
$$

with $\left.\varepsilon=\left(1-a \|^{2} / a_{\perp}\right)^{2}\right)^{1 / 2}$ for oblate spheroids, and as $F=2 \pi a_{\perp}^{2}+2 \pi \frac{a_{\|} a_{\perp}}{\varepsilon} \arcsin \varepsilon$,

$$
\begin{array}{r}
N=\frac{1}{2 \varepsilon^{2}}\left[\sqrt{1-\varepsilon^{2}-} \frac{1-2 \varepsilon^{2}}{\varepsilon} \arcsin \varepsilon\right] \\
\cdot\left[\sqrt{1-\varepsilon^{2}}+(1 / \varepsilon) \arcsin \varepsilon\right]^{-1},
\end{array}
$$

with $\varepsilon=\left(1-a_{\perp}{ }^{2} / a_{\|}{ }^{2}\right)^{1 / 2}$ for prolate spheroids.

An inspection of Eqs. (3.6), (3.8) shows that both forces are proportional to the area $F$ of the particle, but through the quantity $N$ they also depend on its shape. As is already well known from Epstein's ${ }^{2}$ and Waldmann's ${ }^{4,5}$ work on spheres, the frictional force is proportional to the gas pressure; the thermal force is pressure independent.

In a polyatomic gas the heat flux is influenced by a magnetic field. The corresponding change in the thermal force, known as thermomagnetic force, has 
been measured for disks by Adair and coworkers ${ }^{18}$. The first calculation of this force has been performed by Hess ${ }^{17}$ for small $(a \ll l)$ and for large spheres $(a \gg l)$. A subsequent paper will be devoted to the discussion of the thermomagnetic force for spheroids, given by Eqs. (3.7), (3.8).

In a monatomic gas the forces on a particle can easily be calculated for the more general case where the coefficient $\alpha$ of diffuse reflection is different from $1,0<\alpha \leqq 1$, using 4,5

$$
f^{+}=(1-\alpha) f^{\prime}+\alpha f^{\mathrm{M}}\left(n^{+}, T^{+}\right)
$$

instead of (1.3), with the law of specular reflection

$$
f^{\prime}(\boldsymbol{c})=f\left(\boldsymbol{c}^{\prime}\right), \quad \boldsymbol{c}^{\prime}=\boldsymbol{c}-2 \boldsymbol{n} \boldsymbol{n} \cdot \boldsymbol{c}
$$

and

$f^{\mathrm{M}}\left(n^{+}, T^{+}\right)=n^{+}\left(m / 2 \pi k T^{+}\right)^{3 / 2} \exp \left\{-\left(m c^{2} / 2 k T^{+}\right)\right\}$.

Then,

$$
\boldsymbol{K}=-p_{0} \iint \mathrm{d} \sigma[(1-\alpha) \boldsymbol{n} \boldsymbol{n} \cdot \boldsymbol{X}+\alpha / 2(\boldsymbol{X}+\boldsymbol{n} X)]
$$

replaces Eq. (1.9), and especially for spheroidal particles we get

$$
\begin{gathered}
k^{\|}=p_{0} F 2 / \pi[4(1+\alpha \pi / 8)(1-2 \mathrm{~N})-2 \alpha(1-3 N)], \\
k^{\perp}=p_{0} F 2 / \pi[4(1+\alpha \pi / 8) N+\alpha(1-3 N)], \quad(3.12) \\
\text { and } \quad \text { (with } \mathbf{Q}=-\lambda / \bar{c} \nabla T) \\
f^{\|}=F(4 / 5 \pi)[2(1-2 N)-\alpha(1-3 N)], \\
f^{\perp}=F(4 / 5 \pi)[2 N-\alpha / 2(1-3 N)] . \quad(3.13)
\end{gathered}
$$

For a sphere of radius $a$, i. e. $F=4 \pi a^{2}, N=1 / 3$, Eq. (3.12) reduces to Epstein's ${ }^{2}$ result

$$
k^{\|}=k^{\perp}=p_{0} a^{2} 32 / 3(1+\alpha \pi / 8),
$$

and Eq. (3.13) gives Waldmann's formula ${ }^{4,5}$

$$
f^{\|}=f^{\perp}=a^{2} 32 / 15 .
$$

Hence, the frictional force depends on the coefficient $\alpha$, but the thermal force is independent of $\alpha^{4,5}$. However, for $N \neq 1 / 3$ (e.g. $N=0$ for a disk) both forces depend on $\alpha$.

For a gas of rotating molecules the law of specular reflection is more complicated (see e. g. Halbritter's work ${ }^{23}$ ) than for a monatomic gas. Since the complication is due to the behavior of the rotational angular momentum upon reflection it is conceivable that for zero magnetic field Eqs. (3.12), (3.13) are a good approximation for the frictional and the thermal force. The thermomagnetic force, however, might depend more critically on the law of reflection for the angular momentum, i. e. Eq. (3.13) might no longer be the correct expression for $\alpha<1$.

\section{Forces for Spheres, Disks and Rods}

In this section the frictional and the thermal forces (for zero magnetic field) are discussed in particular for spheres, disks and rods. The results derived from Eqs. (3.5) - (3.8) are compared with the expressions available for the hydrodynamic and the free molecular flow regimes. A detailed discussion of the dependence of the thermal force on the orientation of disks and rods relative to the temperature gradient is given. For zero magnetic field, the thermal force is obtained from Eq. (3.7) with

$$
\mathbf{Q}=-\left(\lambda^{\operatorname{trans}} / c\right) \nabla T,
$$

where $\hat{\iota}^{\text {trans }}$ is the translational heat conductivity.

\section{Forces on Spherical Particles}

For $\alpha=1$ both the frictional and the thermal force depend on the properties of the particles only through its surface area, see Eqs. (3.14), (3.15). In the hydrodynamic limit the forces are proportional to the radius ${ }^{1}$

$$
k_{h} \|=k_{h} \perp=\frac{1.5}{2} \pi p_{0} l a,
$$

and, according to Epstein's formula ${ }^{7}$,

$$
f_{h} \|=f_{h} \perp=\frac{15}{2} \pi l a 2 \sigma /(\Lambda+2)
$$

the thermal force depends strongly on the ratio $\Lambda=\lambda_{p} / \lambda$ of the thermal conductivities of the particle

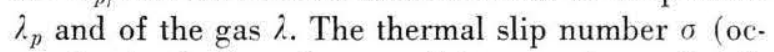
curring in the boundary condition, see Appendix) is approximately equal to $1 / 5$, cf. Reference 12 . Since the thermal force is proportional to the viscosity mean free path $l=\frac{4}{5} \eta \bar{c} p_{0}{ }^{-1}$ it vanishes for high pressures with $1 / p_{0}$. The Stokes force in Eq. (4.1) is independent of pressure.

The hydrodynamic limits of Eqs. (4.1), (4.2) are valid for large particles, i.e. for $a \gg l$, whereas Eqs. (3.14), (3.15) pertain to small particles with $a \ll l$. For a heat conducting gas between parallel plates of distance $D$, Eq. (3.15) applies only if the inequality $a \ll l \ll D$ is fulfilled. The free molecular flow limit is reached for $a \ll D \ll l$, with a thermal force which is proportional to pressure,

$$
f_{k} \|=f_{k}^{\perp}=\frac{3}{4} \pi a^{2}, \quad \boldsymbol{Q}=1 / \bar{c} \boldsymbol{q}_{\text {trans }}^{k},
$$

since this is true for the heat flux

$$
\boldsymbol{q}_{\text {trans }}^{k}=\frac{1}{2} \boldsymbol{g} p_{0} \bar{c} \frac{T_{2}-T_{1}}{T_{0}} \frac{\alpha_{1}{ }^{\mathrm{t}} \alpha_{2}{ }^{\mathrm{t}}}{\alpha_{1}{ }^{\mathrm{t}}+\alpha_{2}{ }^{\mathrm{t}}-\alpha_{1}{ }^{\mathrm{t}} \alpha_{2}{ }^{\mathrm{t}}{ }^{\mathrm{t}} .}
$$

Here, $\boldsymbol{g}$ is the unit vector directed towards the cold plate (at the temperature $T_{1}$ ), and $\alpha_{1}{ }^{\mathrm{t}}, \alpha_{2}{ }^{\mathrm{t}}$ are the 
accommodation coefficients for translational energy at both plates. The expression (4.3) for the thermal force has been derived by Phillips ${ }^{9}$ for a monatomic gas, Brock's formula ${ }^{8}$ contains a numerical error. Phillips ${ }^{9}$ also treats the intermediate regime between the Waldmann limit ${ }^{4,5}$, Eq. (3.15), and the Knudsen limit, Equation (4.3). The decrease of the thermal force from the Waldmann limit is due to the finite plate spacing $D$. At least for small values of $l / D$ this decrease can be obtained from Eq. (3.15) by taking into account the reduction of the temperature gradient due to the temperature jump ${ }^{24}$ :

$$
\begin{array}{r}
\nabla T=(\nabla T)_{0}\left(1+2 l / D C_{\mathrm{t}}\right)^{-1}, \text { i. e. } \\
\boldsymbol{K}^{\text {th }}=-\frac{32}{15} a^{2} \frac{\lambda^{\text {trans }}}{\bar{c}}(\nabla T)_{0}\left(1+2 l / D C_{t}\right)^{-1} .
\end{array}
$$

Here $(\nabla T)_{0}$ is the temperature gradient for $l / D \rightarrow 0$, the temperature jump coefficient ${ }^{24} C_{\mathrm{t}}$ is of the order of 2 to 3 .

\section{Forces on Spheroidal Particles}

In the case of a sphere the thermal force has the same direction as the negative temperature gradient, the frictional force, respectively, is parallel to the flow velocity. In contradistinction, for a spheroidal particle (e.g. a disk or a rod) the thermal force has components parallel to $-\nabla T$ and parallel to the symmetry axis $\boldsymbol{s}$, i. e. in general the force has components parallel as well as perpendicular to $-\nabla T$. The unit vector parallel to $-\nabla T$ is denoted by $\boldsymbol{g}$, then $\boldsymbol{Q}=|\boldsymbol{Q}| \boldsymbol{g}$. Both, $\boldsymbol{s}$ and $-\boldsymbol{s}$ are equivalent for the characterization of the symmetry axis of the spheroid. Now, by $\boldsymbol{s}$ we specifically denote that vector which encloses an acute angle $\chi$ with $\boldsymbol{q}$, i. e. $\sigma \leqq \chi \leqq \pi / 2$. Then, the unit vector $\boldsymbol{e}$ perpendicular to $\boldsymbol{g}$ is defined (see Figure $1 \mathrm{a}$ ) :

$$
\begin{array}{r}
\boldsymbol{e}=\frac{1}{\sin \chi}(\boldsymbol{s}-\cos \chi \boldsymbol{g}), \boldsymbol{e} \cdot \boldsymbol{g}=0, \\
\boldsymbol{s} \cdot \boldsymbol{g}=\cos \chi, \boldsymbol{s} \cdot \boldsymbol{e}=\sin \chi .1)
\end{array}
$$

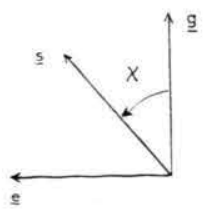

(a)
Fig. 1. Components of the thermal force for spheriodal particles. (a) Definition of the unit vector $\boldsymbol{e}$ according to Eq. (5.1); $\mathbf{g}$ is parallel to $-\nabla T, \boldsymbol{s}$ is the symmetry axis of spheroid. Thermal force in the Waldmann limit for

(b) a disk from Eq. (5.5), and (c) a rod from Eq. (5.12).
The thermal force from Eqs. (3.7), (3.8) can be rewritten as

$$
\begin{gathered}
\boldsymbol{K}^{\mathrm{th}}=\boldsymbol{K}_{e}+\boldsymbol{K}_{g}, \\
\boldsymbol{K}_{e}=\boldsymbol{e}|\boldsymbol{Q}|\left(f^{\|}-f^{\perp}\right) \sin \chi \cos \chi, \\
\boldsymbol{K}_{g}=\boldsymbol{g}|\boldsymbol{Q}|\left[f^{\|}-\left(f^{\|}-f^{\perp}\right) \sin ^{2} \chi\right] .
\end{gathered}
$$

For $\chi=0$ and $\chi=\pi / 2$ the perpendicular component $\boldsymbol{K}_{e}$ vanishes, the parallel component takes on the values $\boldsymbol{K}_{g}=\boldsymbol{g}|\boldsymbol{Q}| f^{\|}$, and $\boldsymbol{K}_{g}=\boldsymbol{g} \boldsymbol{Q} \mid f^{\perp}$, respectively. In particular, if $f^{\|}$is larger than $f^{\perp}$ the component $\boldsymbol{K}_{g}$ decreases with increasing angle $\chi$, and $\boldsymbol{K}_{e}$ has the direction $\boldsymbol{e}$, reaching its maximum value $\frac{1}{2}|\boldsymbol{Q}|\left(f^{\|}-f^{\perp}\right)$ for $\chi=\pi / 4$. For $f^{\|}<f^{\perp}$ the component $\boldsymbol{K}_{e}$ has the direction - $\boldsymbol{e}$. In other words: Let $\boldsymbol{s}$ and $\boldsymbol{g}$ enclose an acute angle, then $\boldsymbol{K}_{e}$ and $\boldsymbol{s}$ enclose an acute (an obtuse) angle, if $f^{\|}$is larger (smaller) than $f \perp$.

The same considerations apply to the frictional force, with $\boldsymbol{g}$ parallel to the flow velocity $\boldsymbol{v}$.

\section{(a) D is k s}

A disk of radius $a_{\perp}$ and thickness $2 a_{\|}$in the center is the limit of an oblate spheroid for $a_{\|} / a_{\perp} \rightarrow 0$. With $\mathrm{F}=2 \pi a_{\perp}{ }^{2}$ and $N=0$ from (3.10), Eqs. (3.6), (3.8) reduce to

$k^{\|}=8 a_{\perp}^{2}(1+\pi / 4) p_{0}, \quad k^{\perp}=4 a_{\perp}^{2} p_{0}=0.280-k^{\|}$,

$$
f^{\|}=\frac{8}{5} a_{\perp}^{2}, \quad f^{\perp}=\frac{4}{5} a_{\perp}^{2}=0.5 f^{\|} .
$$

In the Knudsen limit the thermal force is determined by $\boldsymbol{q}_{\text {trans }}^{k}$ from Eq. (4.4) and by

$$
f_{k}^{\|}=\frac{1}{2} a_{\perp}^{2} \pi, f_{k}^{\perp}=a_{\perp}^{2}=0.636 f_{k}^{\|},
$$

the expression for $f_{k} \|$ has already been given by Knudsen ${ }^{10}$. In all the cases of Eqs. (5.4) - (5.6) the component $\boldsymbol{K}_{g}$ has its maximum value for $\chi=0$, i. e. if the temperature gradient (or the flow velocity) is parallel to the normal of the disk. Correspondingly, the component $\boldsymbol{K}_{e}$ has the same direction as $\boldsymbol{e}$ (see Figure $1 \mathrm{~b}$ ).

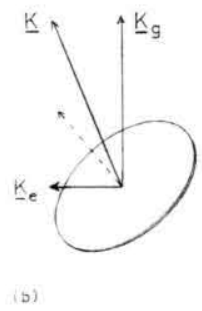

Fig. 1 b.

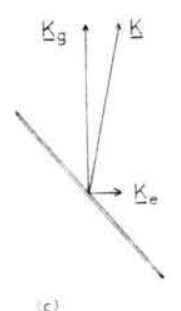

Fig. 1 c. 
The situation is more complicated in the hydrodynamic limit (see Appendix) :

$$
\begin{gathered}
k_{\mathrm{h}}^{\|}=20 a_{\perp} l p_{0}, k_{\mathrm{h}}^{\perp}=\frac{40}{3} a_{\perp} l p_{0}, \\
f_{\mathrm{h}}^{\|}=10 \pi a l \frac{\sigma}{\left(A+\frac{a}{a_{\perp}} \frac{\pi}{2}\right)}, \\
f_{\mathrm{h}}^{\|}=\frac{40}{3} a_{\perp} l \frac{\sigma}{\left(1+\frac{\pi}{4} \frac{a_{\|}}{a_{\perp}} A\right)} .
\end{gathered}
$$

For the frictional force ${ }^{1} k_{\mathrm{h}} \| \mid k_{\mathrm{h}} \perp=\frac{3}{2}$ applies, for the thermal force, however, this ratio depends strongly of the relative heat conductivity $\Lambda=\lambda_{\mathrm{p}} / \lambda$. In the limit $A \ll a_{\|} / a_{\perp} \pi / 2 \rightarrow 0$ (which is probably rather unrealistic) one has again the ratio $3 / 2$ :

$$
f_{\mathrm{h}}{ }^{\|}=20 a \perp l \sigma=\frac{3}{2} f_{\mathrm{h}} \perp .
$$

For all realistic cases $A \gg a_{\|} / a_{\perp} \pi / 2$ applies, then we have

$$
\begin{aligned}
& f_{\mathrm{h}}\|==10 \pi a\| l \frac{\sigma}{A}, \\
& f_{\mathrm{h}} \| \mid f_{\mathrm{h}} \perp=\frac{3}{4} \pi \frac{a \|}{a_{\perp}}\left(\frac{1}{\Lambda}+\frac{\pi}{4} \frac{a_{\|}}{a_{\perp}}\right),
\end{aligned}
$$

i. e. $f_{\mathrm{h}} \|$ is inversely proportional to $A$, and is (due to $a \| / a_{\perp} \rightarrow 0$ ) in any case much smaller than $f_{\mathrm{h}} \perp$. Consequently $\boldsymbol{K}_{e}$ has now the direction $-\boldsymbol{e}$, and already for very small angles $\chi$ the component $\boldsymbol{K}_{g}$ is essentially proportional to $\sin ^{2} \chi$ :

$$
\boldsymbol{K}_{g} \approx \boldsymbol{g}|\boldsymbol{Q}| f_{\mathrm{h}}^{\perp} \sin ^{2} \chi .
$$

This strange behavior is due to the fact that, in the hydrodynamic limit, the thermal force is proportional to the temperature difference over the disk, hence (for $A \neq 0$ ) proportional to $a \|$ and $A^{-1}$ for $f_{\mathrm{h}} \| 7,11$, and proportional to $a_{\perp}$ (and very weakly dependent on $\Lambda$ ) for $f_{\mathrm{h}} \perp{ }^{11}$, respectively. It is rather doubtful, however, whether or not these limits can be met experimentally.

\section{(b) Rods}

For a very long prolate spheroid $\left(a_{\perp} / a_{\|} \rightarrow 0\right)$ Eq. (3.11) gives $F=\pi^{2} a_{\|} a_{\perp}, N=\frac{1}{2}$. The forces on such a rod (length $2 a_{\|}$, radius $a_{\perp}$ ) are obtained from Eqs. (3.6), (3.8) :

$$
\begin{aligned}
& k^{\|}=2 \pi a_{\|} a_{\perp} p_{0},(5.11) \\
& k^{\perp}=3 \pi a_{\|} a_{\perp}(1+\pi / 6) p_{0}=2.285 k \|, \\
& f^{\|}=\frac{2}{5} \pi a_{\|} a_{\perp}, \quad f^{\perp}=\frac{3}{5} \pi a_{\|} a_{\perp}=\frac{3}{2} f^{\|} .
\end{aligned}
$$

Here, for both forces the component $\boldsymbol{K}_{g}$ has its maximum value for $\chi=\pi / 2$, i. e. if the axis of the $\operatorname{rod}$ is perpendicular to $\boldsymbol{g}$. As a consequence, $\boldsymbol{K}_{e}$ has the same direction as $-\boldsymbol{e}$ (see Figure $1 \mathrm{c}$ ). The same qualitative behavior is found for the frictional force in the hydrodynamic limit ${ }^{1}$ :

$$
k_{\mathrm{h}}\left\|=\frac{5 \pi a_{\|} l}{\ln \left(a_{\|} / a_{\perp}\right)} p_{0}, k_{\mathrm{h}} \perp=2 k_{\mathrm{h}}\right\| .
$$

For the thermal force, however, the situation is more complicated:

$$
f_{\mathrm{h}}\left\|=\frac{5 \pi a_{\|} l}{\ln \left(a_{\|} / a_{\perp}\right)} \sigma, \quad f_{\mathrm{h}} \perp=\frac{2}{\Lambda+1} f_{\mathrm{h}}\right\| .
$$

In particular, $f_{\mathrm{h}} \|$ is independent of $A$, whereas $f_{\mathrm{h}} \perp$ is proportional to $(\Lambda+1)^{-1}$, for moderate values of $A$ both are of the same order of magnitude. Notice, $f_{\mathrm{h}} \perp \approx 2 f_{\mathrm{h}} \|$ for $A \ll 1, f_{\mathrm{h}} \perp=f_{\mathrm{h}} \|$ for $A=1$, and $f_{\mathrm{h}} \perp<f_{\mathrm{h}} \|$ for $A>1$.

Hence, the component $\boldsymbol{K}_{e}$ is parallel to $-\boldsymbol{e}$ for $A<1$, and parallel to $\boldsymbol{e}$ for $A>1$, for $A=1$ it is not present at all.

In this paper, the "Waldmann limits" (i. e. $a \ll l \ll D$ ) of the frictional and thermal forces have been calculated for spheroidal particles suspended in a polyatomic gas. The results have been compared to the corresponding expressions in the hydrodynamic limit $(l \ll a \ll D)$, and, for the thermal force, also to the formulae available in the free molecular flow limit. In the slip flow regime $(l \ll a$ up to $l \lesssim x$ ) a lot of work has been done for spheres, see e.g. References 12-15. Calculations for the thermal force on a sphere in the transition regime from the Waldmann limit to the Knudsen limit are due to Phillips ${ }^{9}$. For nonspherical particles, how ever, neither of these regions has been treated up to now.

\section{Appendix}

\section{The Forces in the Hydrodynamic Limit}

For convenience, some of the well known hydrodynamic expressions are stated. The force on the ellipsoid

$$
\sum_{i=1}^{3} x_{i}{ }^{2} / a_{i}^{2}=1
$$

is written as

$$
\mathbf{K}=6 \pi \eta \sum_{i=1}^{3} R_{i} \boldsymbol{e}_{i} \boldsymbol{e}_{i} \cdot \boldsymbol{V},
$$


where $\boldsymbol{e}_{1}, \boldsymbol{e}_{2}, \boldsymbol{e}_{3}$ form an orthonormal basis of three-dimensional space. For the frictional force $K^{\mathrm{v}}$, the vector $\boldsymbol{V}$ is equal to the gas velocity $\boldsymbol{v}_{\infty}$ far away from the particle, $\boldsymbol{V}=\boldsymbol{v}_{\infty}$. And, according to Lamb ${ }^{1}$, the length $R_{i}$ has to be replaced by

$$
R_{i}^{\mathrm{v}}=\frac{\frac{8}{3} a_{1} a_{2} a_{3}}{\chi+\alpha_{i} a_{i}^{2}},
$$

with

$$
\begin{aligned}
& \chi=a_{1} a_{2} a_{3} \int_{0}^{\infty} \mathrm{d} \lambda\left[\left(a_{1}{ }^{2}+\lambda\right)\left(a_{2}{ }^{2}+\lambda\right)\left(a_{3}{ }^{2}+\lambda\right)\right]^{-1 / 2}, \\
& \alpha_{i}=a_{1} a_{2} a_{3} \int_{0}^{\infty} \mathrm{d} \lambda\left(a_{i}^{2}+\lambda\right)^{-1}\left[\left(a_{1}^{2}+\lambda\right)\right. \\
& \left.\cdot\left(a_{2}{ }^{2}+\lambda\right)\left(a_{3}{ }^{2}+\lambda\right)\right]^{-1 / 2}
\end{aligned}
$$

Lamb's method ${ }^{1}$ can also be applied for the calculation of the thermal force $\boldsymbol{K}^{\text {th }}$, using the boundary condition of thermal slip in the form

$$
\boldsymbol{v}^{\tan }=\sigma \frac{\lambda^{\operatorname{trans}}}{p_{0}}(\nabla T)^{\tan } .
$$

Here $\lambda^{\text {trans }}$ is the translational heat conductivity of the gas, and $\sigma$ is the thermal slip number. Typically, $\sigma$ is approximately equal to $1 / 5$, the number first given by Maxwell (see also Ref. 12). Then, in Eq. (A1) the vector $\boldsymbol{V}$ has to be replaced by

$$
\boldsymbol{V}=-\sigma\left(\lambda^{\text {trans }} / p_{0}\right)(\nabla T)_{\infty},
$$

and the length $R_{i}$ by

$$
R_{i}^{\text {th }}=R_{i}^{v} \frac{2-\alpha_{i}}{2-\alpha_{i}(1-\Lambda)} .
$$

By $(\nabla T)_{\infty}$ the temperature gradient in the gas, far away from the ellipsoid is denoted, $A=\lambda_{p} / \lambda$ is the ratio of the heat conductivities of the particle $\lambda_{p}$ and of the gas $\lambda$. Notice, $R_{i}^{\text {th }} \rightarrow R_{i}^{\mathrm{v}}$ for either $\alpha_{\mathrm{i}} \rightarrow 0$ or $\Lambda \rightarrow 0$.

For an oblate spheroid the thermal force has been calculated by Sexl ${ }^{11}$; in particular, he treated the case that $(\nabla T)_{\infty}$ is parallel to $\boldsymbol{e}_{1}$, and expressed $\boldsymbol{K}^{\text {th }}$ by $R_{1}{ }^{\mathrm{v}}$ and by the temperature difference

$$
\delta T=\frac{4 a_{1}}{2-\alpha_{1}(1-\Lambda)} \boldsymbol{e}_{1} \cdot(\nabla T)_{\infty}
$$

1 H. Lamb, "Hydrodynamics", Dover Publication, New York 1945 (Sixth Edition), p. 604. See also J. Happel and H. Brenner, "Low Reynolds Number Hydrodynamics", Prentice Hall, Englewood Cliffs, New Jersey 1965.

2 P. S. Epstein, Phys. Rev. 23, 710 [1924].

3 A. Einstein, Z. Physik 27, 1 [1924]. between the poles $x_{1}=a_{1}, x_{2}=x_{3}=0$ and $x_{1}=-a_{1}$, $x_{2}=x_{3}=0$ of the ellipsoid. A different approach for the thermal force on a flat disk is due to Epstein ${ }^{7}$.

If the ellipsoid has rotational symmetry about the axis $\boldsymbol{s} \equiv \boldsymbol{e}_{1}$, with $\boldsymbol{a}_{\|} \equiv a_{1}, a_{\perp} \equiv a_{2}=a_{3}$, and $R_{\|} \equiv R_{1}, R_{\perp} \equiv R_{2}=R_{3}$. Equation (Al) can be rewritten in the form

$$
\boldsymbol{K}=6 \pi \eta\left[R_{\|} \boldsymbol{s} \boldsymbol{s} \cdot \boldsymbol{V}+R_{\perp}(\boldsymbol{V}-\boldsymbol{s} \boldsymbol{s} \cdot \mathbf{V})\right] .
$$

Analytic expressions for $\chi$ and $\alpha_{\|} \equiv \alpha_{1}, \alpha_{\perp} \equiv \alpha_{2}=\alpha_{3}$ can be found in a paper by Gans ${ }^{19}$. The result for oblate spheroids is

$$
\begin{aligned}
& \chi=2 a_{\|} a_{\perp}(1 / \varepsilon) \arcsin \varepsilon, \\
& \alpha_{\|}=\left(2 / \varepsilon^{2}\right)\left[1-\left(\sqrt{1-\varepsilon^{2} / \varepsilon}\right) \arcsin \varepsilon\right], \\
& \alpha_{\perp}=\left(1 / \varepsilon^{2}\right)\left[\left(\sqrt{1-\varepsilon^{2}} / \varepsilon\right) \arcsin \varepsilon-\left(1-\varepsilon^{2}\right)\right],
\end{aligned}
$$

with $\varepsilon=\left(1-a \|^{2} / a_{\perp}{ }^{2}\right)^{1 / 2}$. For prolate spheroids we have $\varepsilon=\left(1-a_{\perp}{ }^{2} / a_{\|} \|^{2}\right)^{1 / 2}$ and

$$
\begin{aligned}
\chi & =a \|^{2} \frac{1-\varepsilon^{2}}{\varepsilon} \ln \frac{1+\varepsilon}{1-\varepsilon}, \\
\alpha_{\|} & =\frac{1-\varepsilon^{2}}{\varepsilon^{2}}\left[\frac{1}{\varepsilon} \ln \frac{1+\varepsilon}{1-\varepsilon}-2\right], \\
\alpha_{\perp} & =\frac{1}{\varepsilon^{2}}-\frac{1-\varepsilon^{2}}{2 \varepsilon^{3}} \ln \frac{1+\varepsilon}{1-\varepsilon} .
\end{aligned}
$$

In the case of a sphere of radius $a$ Eqs. (A5), (A6) reduce to

$$
\chi=2 a^{2}, \alpha_{\|}=\alpha_{\perp}=2 / 3 .
$$

In particular, for a disk $\left(a_{\|} / a_{\perp} \rightarrow 0\right) \mathrm{Eq}$. (A5) yields $\chi=\pi a_{\|} a_{\perp}, \quad \alpha_{\|}=2-\pi\left(a_{\|} / a_{\perp}\right), \quad \alpha_{\perp}=\frac{1}{2} \pi a_{\|} / a_{\perp}$

and Eq. (A6) gives for a rod $\left(a_{\perp} / a_{\|} \rightarrow 0\right)$

$$
\begin{gathered}
\chi=2 a_{\perp}^{2} \ln \left(a_{\|} / a_{\perp}\right), \quad \alpha_{\|=2}\left(a_{\perp}^{2} / a_{\|}^{2}\right) \ln \left(a_{\|} / a_{\perp}\right), \\
\alpha_{\perp}=1-\left(a_{\perp}^{2} / a_{\|}^{2}\right) \ln \left(a_{\|} / a_{\perp}\right) .
\end{gathered}
$$

\section{Acknowledgements}

This research was supported by the Robert A. Welch Foundation, Houston, Texas. The author would like to express appreciation to Professor T. W. Adair, III for stimulating discussions and useful comments. Thanks are also due to Dr. L. A. Davis for his valuable assistance.

4 L. Waldmann, Z. Naturforsch. 14 a, 589 [1959].

5 L. Waldmann, in Proc. 2nd Int. Symp. Rarefied Gas Dynamics, Berkeley 1960, ed. L. Talbot, Academic Press, New York 1961, p. 323.

6 G. Hettner, Z. Physik 37, 179 [1926].

7 P. S. Epstein, Z. Physik 54, 537 [1929]. 
8 J. R. Brock, J. Coll. Interface Sci. 25, 564 [1967].

9 W. F. Phillips, Phys. Fluids 15, 999 [1972].

10 M. Knudsen, Ann. Physik 32, 809 [1910]. - M. v. Smoluchowski, Ann. Physik 34, 182 [1911].

11 Th. Sexl, Z. Physik 52, 249 [1928].

${ }^{12}$ L. Waldmann and K. H. Schmitt, in "Aerosol Science", ed. C. N. Davies, Academic Press, London 1966, Chap. VI, p. 137.

13 S. Jacobsen and J. R. Brock, J. Coll. Sci. 20, 544 [1965].

14 H. A. Dwyer, Phys. Fluids 10, 976 [1967].

15 H. Vestner, M. Kübel, and L. Waldmann, in Proc. 7th Int. Symp. Rarefied Gas Dynamics, Pisa 1970, ed. D. Dini, Academic Press, New York, in press.

16 J. J. M. Beenakker and F. R. McCourt, Ann. Rev. Phys. Chem. 21, 47 [1970].

17 S. Hess, Z. Naturforsch. 27 a, 366 [1972].
18 M. E. Larchez and T. W. Adair, III, Phys. Rev. Letters 25, 21 [1970] ; Phys. Rev. A 3, 2052 [1971]. - J. Taboada, J. Chem. Phys., to be published - L. A. Davis, J. Chem. Phys., to be published.

19 R. Gans, Ann. Physik 86, 628 [1928].

20 S. Hess and L. Waldmann, Z. Naturforsch. 21 a, 1529 [1966] ; 23 a, 1893 [1968]; 26 a, 1057 [1971]. - H. H. Raum and W. E. Köhler, Z. Naturforsch. 25 a, 1178 [1970].

21 L. Waldmann, Z. Naturforsch. 12 a, 660 [1957]; 13 a, 609 [1958]. - R. F. Snider, J. Chem. Phys. 32, 1051 [1960].

22 S. Hess, Z. Naturforsch. 28 a, 1531 [1973].

23 J. Halbritter, Thesis, Erlangen 1972; Z. Naturforsch., to be published.

24 M. v. Smoluchowski, Ann. Physik 35, 983 [1911]. - See also S. A. Schaaf, "Mechanics of Rarefied Gases", in Handbuch der Physik (ed. S. Flügge), Vol. 8/2, p. 591, Springer-Verlag, Berlin 1963. 